\title{
Does Low Intracellular pH Stop the Motion of the Bulla Circadian Pacemaker?
}

\author{
Sat Bir S. Khalsa, ${ }^{1}$ Martin R. Ralph, ${ }^{2}$ and Gene D. Block' \\ 'Department of Biology, University of Virginia, Charlottesville, Virginia 22901 and ${ }^{2}$ Department of Psychology, University of \\ Toronto, Toronto, Ontario, Canada M5S 1A1
}

The eye of the mollusk Bulla has proven itself useful as an in vitro neural circadian pacemaker. Here, we report that treatments applied to lower intracellular $\mathrm{pH}$ may stop the motion of this circadian pacemaker in a phase-dependent manner.

Lowering the extracellular $\mathrm{pH}$ of the artificial seawater bath to 6.9, or application of the stilbene derivatives 4-acetamido$4^{\prime}$-isothiocyanostilbene-2,2' -disulfonic acid (SITS) or 4,4-diisothiocyanostilbene-2,2'-disulfonic acid (DIDS), abolishes the circadian rhythm in optic nerve compound action-potential frequency. Because these treatments are known to lower intracellular $\mathrm{pH}$, these data suggest that the pacemaker may be inhibited by low intracellular pH.

In order to assess the state of the pacemaker during low extracellular $\mathrm{pH}$ treatment, pulses of seawater at $\mathrm{pH} 6.8$ were applied, and the phase of the rhythm subsequent to the pulse was observed. All pulses started $1 \mathrm{hr}$ after subjective dusk [circadian time (CT) 13] and were applied to eyes in constant darkness; pulse lengths varied from 4 to $47 \mathrm{hr}$ for different preparations. The phases of the eye rhythms following pulses that ended before subjective dawn (about CT 24) were not different from untreated preparations. However, for pulses longer than $11 \mathrm{hr}$ and therefore ending after subjective dawn, the subsequent phase of the rhythm was a function of the ending time of the pulse. These data suggest that the pacemaker's motion was stopped at dawn during the lowpH treatment and resumed following restoration of normal pH.

To distinguish between phase and duration dependence of this effect in the above experiment, phase shifts were obtained to 14-hr pulses of pH 6.8 seawater applied at three different phases. These phase shifts were of significantly different magnitude, suggesting that the low-pH treatment must include a critical phase near subjective dawn in order for pacemaker motion to be stopped.

Large phase shifts also followed long pulses of the weak acid salicylate, which is known to lower intracellular pH. The phase shifts induced by 23-hr pulses were concentration dependent and exhibited a sharp change in magnitude from advances of $2 \mathrm{hr}$ to delays of 10-12 hr over a narrow range of salicylate concentration.

\footnotetext{
Received Oct. 29, 1990; revised Apr. 1, 1991; accepted Apr. 5, 1991.

This work was supported by NIH Grant NS15264 to G.D.B. and by National Research Service Award NS09621 to S.B.S.K.

Correspondence should be addressed to Dr. Gene D. Block, Department of Biology, Gilmer Hall, University of Virginia, Charlottesville, VA 22901.

Copyright (C) 1991 Society for Neuroscience 0270-6474/91/112672-08\$03.00/0
}

We believe that a reduction in intracellular $\mathrm{pH}$ is responsible for our observations; however, a direct measurement of intracellular pH is necessary to confirm this conclusion.

Circadian rhythms are ubiquitous in eukaryotic organisms. In the animal kingdom, the cells responsible for generating the circadian timing are often located in neural and endocrine structures: the retina in some amphibians and mollusks, the optic lobe in the cockroach, the pineal in some birds and lizards, and the suprachiasmatic nucleus of the hypothalamus in mammals (Jacklet, 1984, 1989; Takahashi et al., 1989). The molluscan eye has been particularly useful as an in vitro preparation of a neural circadian pacemaker (Jacklet, 1989).

The basal retinal neurons in the eye of the mollusk Bulla gouldiana generate a rhythm of compound action potentials (CAPs) in the optic nerve that can be recorded extracellularly for up to $7 \mathrm{~d}$ in artificial seawater (ASW; Block and Wallace, 1982). Previous studies have identified elements that are involved in both the "input" and the "output" pathways to this pacemaker. On the input pathway, light pulses are capable of phase shifting the rhythm in a phase-dependent manner consistent with other circadian systems. The phase-shifting action of light is due to a light-induced depolarization and requires a concomitant transmembrane flux of calcium through voltagedependent calcium channels (McMahon and Block, 1987; Khalsa and Block, 1988). On the output pathway, the circadian rhythm in CAP activity appears to be generated by a rhythm in membrane potential of the basal retinal neurons (McMahon et al., 1984), which in turn may be driven by rhythmic conductance changes hypothesized to be changes in potassium conductance (Ralph and Block, 1990). Aside from a sequential analysis of elements comprising the input and output pathways of the pacemaker, other experimental approaches to elucidation of the underlying circadian pacemaker are also possible.

Although it is widely accepted that the intracellular (cytosolic) $\mathrm{pH}$ of animal cells other than erythrocytes is regulated to values more basic than predicted by an equilibrium distribution of hydrogen ions (Roos and Boron, 1981), the view that intracellular $\mathrm{pH}$ levels can be assumed to be invariant and of no regulatory significance is not tenable (Busa and Nuccitelli, 1984). Changes in intracellular $\mathrm{pH}$ levels appear to accompany numerous cellular processes that exhibit their own characteristic $\mathrm{pH}$ sensitivities. For example, intracellular $\mathrm{pH}$ has been observed to function synergistically with second-messenger systems in stimulus-response coupling (Busa and Nuccitelli, 1984; Busa, 1986), as a potential regulator of fertilization and protein synthesis (Winkler, 1982), and even as a pacemaker in driving the cell cycle (Aerts et al., 1985). 
Characterization of a circadian pacemaker's $\mathrm{pH}$ sensitivity, by manipulating intracellular $\mathrm{pH}$, may provide useful information toward an analysis of the pacemaker's underlying mechanism. It is also conceivable that intracellular $\mathrm{pH}$ may play an active role in circadian rhythm generation. We have assessed the sensitivity and/or role of intracellular $\mathrm{pH}$ in the circadian timing process of the Bulla pacemaker. In this article, we report that treatments applied in order to lower intracellular $\mathrm{pH}$, especially the lowering of extracellular $\mathrm{pH}$, appear to stop the motion of the circadian pacemaker. Furthermore, the effect of the treatment is phase dependent.

\section{Materials and Methods}

Bulla gouldiana werc obtaincd from Marinus Inc. and maintaincd in a temperature-controlled seawater tank at $15^{\circ} \mathrm{C}$. Animals were exposed to a light cycle of $12 \mathrm{hr}$ of light and $12 \mathrm{hr}$ of darkness (LD 12:12) for at least 1 week prior to experimental setup. Two hours before the onset of darkness, animals were immobilized with an injection of $10 \mathrm{ml}$ isotonic $\mathrm{MgCl}_{2}$, then placed on ice for dissection.

For extracellular recordings, both eyes including the optic nerves were removed from each animal and placed in separate dishes of artificial seawater (ASW; $20 \mathrm{ml} /$ dish). The composition of ASW was $395 \mathrm{~mm}$ $\mathrm{NaCl}, 10 \mathrm{mM} \mathrm{KCl}, 10 \mathrm{~mm} \mathrm{CaCl}, 50 \mathrm{~mm} \mathrm{MgCl}, 28 \mathrm{~mm} \mathrm{Na}_{2} \mathrm{SO}_{4}, 30$ $\mathrm{mM}$ HEPES buffer, 100,000 $\mathrm{U}$ penicillin/liter, and 100,000 $\mu \mathrm{g}$ streptomycin/liter. The $\mathrm{pH}$ was adjusted to 7.8 for normal ASW or lower with $\mathrm{HCl}$. The optic nerve from each eye was pulled up into an ASWfilled micropipette suction electrode mounted on a recording dish, which was then placed in a light-tight recording chamber and maintained at $15^{\circ} \mathrm{C}$ for at least $6 \mathrm{~d}$.

Optic nerve compound action potential activity was recorded on a Grass polygraph and was also fed to an analog-to-digital converter where a computer program counted the CAPs into $15-\mathrm{min}$ bins. The phase reference point for the daily peaks of activity for each pair of eyes was determined by calculating the time of occurrence of the half-maximum spike frequency on the rising phase of each cycle of activity. An acceptable cycle of activity was defined as one that had at least one bin of eight events (a minimum frequency of 16 impulses per $0.5 \mathrm{hr}$ ). The average peak impulse frequency was calculated as the average of the counts from those 30 -min bins with the highest values in a circadian cycle over the first four cycles of activity.

Clock time (24-hr day), rather than circadian time (CT), was used in the measurement of the phases of CAP activity cycles and the timing of treatments. Therefore, phases and times reported in circadian time (CT) in this study are slightly later than true circadian time because the period of the in vitro Bulla ocular rhythm is, on average, slightly less than $24 \mathrm{hr}$.

Three types of experiments were performed.

(1) Continuous treatment experiments were performed to evaluate changes in period and/or wave form. Eyes were set up in vitro in ASW at pH 7.8 at CT 12 (subjective dusk). One hour later, at CT 13, the experimental solution was applied. One eye from each animal served as the control for the contralateral one. The preparations were then left undisturbed for the remainder of the experiment.

(2) Phase-shifting experiments were performed, in which contralateral eyes were run as untreated controls. Eyes were set up in vitro in ASW at pH 7.8 at CT 12 (subjective dusk). Pulse treatments were applied either before the first peak of activity or after the first peak and prior to the second peak of activity. In the former case, the phase shift between CAP activity rhythms for experimental and control eyes was taken as the phase difference on the fourth cycle of activity of the control eye. In the latter case, the phase shift was taken as the phase difference on the third cycle (the second cycle after the treatment), minus the phase difference from the first (pretreatment) cycle. In order for data to contribute to these experiments, both eyes were required to complete four cycles of activity.

(3) Long-duration pulse experiments of $\mathrm{pH} 6.8$ artificial seawater were performcd, in which the phases of up to fivc subsequent cycles of activity were recorded. Eyes were set up in vitro in ASW at pH 7.8 at CT 12 (subjective dusk). All pulses were begun at CT 13, $1 \mathrm{hr}$ later, and pulse lengths ranged from 4 to $47 \mathrm{hr}$. At least two eyes were evaluated for each pulse length, and eyes from the same animal were never subjected to the same pulse length.
For each solution exchange, at least $95 \%$ of the volume of the dish was first removed, and $20 \mathrm{ml}$ of the exchanging solution (maintained at $15^{\circ} \mathrm{C}$ ) was infused. One exchange was made for drug treatments, whereas five exchanges were used for ASW at different $\mathrm{pH}$ values. For either phase-shifting or long-duration pulses, five washes of ASW at $\mathrm{pH}$ 7.8 were made at the end of the treatment. In the case of hydrophobic compounds, three exchanges of ASW with dimethyl sulfoxide (DMSO)ASW were followed by two exchanges of ASW at the end of the pulse.

Hydrophobic compounds were first dissolved in DMSO, and these stock solutions were then dissolved in ASW so that DMSO was at a final concentration of $0.05 \%$. All other compounds were added to ASW directly. Salicylate and 4,4-diisothiocyanostilbene-2,2'-disulfonic acid (DIDS) were obtained from Sigma Chemical Company, and 4-acetamido-4'-isothiocyanostilbene-2,2'-disulfonic acid (SITS) was obtained from Research Organics.

\section{Results}

\section{Effects of continuously applied treatments}

Reducing the pH of the ASW from the normal value of 7.8 to $7.3(N=4)$ yielded minor changes in the waveform of the circadian rhythm as compared to rhythms from control eyes from the same animal at $\mathrm{pH} 7.8$ (Fig. 1A). The average peak impulse frequency was reduced by $14 \%( \pm 25 \% ; 95 \%$ confidence interval) relative to controls, though this was statistically insignificant; period was unaffected. At pH $7.1(N=4)$, the average peak impulse frequency was reduced by $32 \%( \pm 24 \%)$, and the period and/or phase of the rhythms appeared unstable as compared with controls (Fig. $1 B$ ). At pH $7.0(N=4)$, the average peak impulse frequency was substantially reduced ( $72 \pm 24 \%$ ), and impulse activity was evident during the usually silent phases of the circadian cycle (Fig. 1C). For all four eyes, the phase of the first cycle of activity was similar to that of controls, whereas the phase appeared advanced with respect to controls for subsequent cycles (see Fig. $1 C$ ). When the extracellular $\mathrm{pH}$ was reduced to $6.9(N=4)$, all eyes tested failed to exhibit circadian rhythmicity. Only sustaincd low-frcquency CAP activity was apparent (Fig. 1D). In three of the four eyes tested, CAP activity began in phase with the first peak of activity of the control eye and became arrhythmic thereafter. Impulse activity was completely suppressed at pH 6.8 (see experiments below) at least over the first cycle.

Inhibition of the cellular $\mathrm{pH}$-regulating mechanism was also used as a technique for lowering intracellular $\mathrm{pH}$ (Ganz et al., 1989). Two acid extrusion inhibitors, the disulfonic stilbene derivatives DIDS (30 $\mu \mathrm{M} ; N=2)$ and SITS $(50 \mu \mathrm{M} ; N=2)$, were very effective at yielding CAP activity devoid of circadian modulation. Both DIDS (Fig. $1 E$ ) and SITS (Fig. $1 F$ ) yielded activity records in which CAP activity began at the beginning of the first cycle of activity, and continued at a constant frequency, as observed for the experiment with pH 6.9 above (Fig. 1D). Because this experiment used DMSO as a carrier for these hydrophobic compounds, control expcriments were run with $0.05 \%$ DMSO in ASW ( $N=4$; data not shown); the circadian rhythms of the treated eyes were not different from those of untreated controls.

Attempts to observe arrhythmicity by lowering intracellular $\mathrm{pH}$ with a weak acid were unsuccessful. Salicylate (Na salt) was added to ASW and was applied continuously starting at CT 13 at concentrations of $1,3,4,5.5$, and $6 \mathrm{~mm}(N=2$ each) and 5 $\operatorname{mm}(N=4)$. Al 1 mm salicylate, no difference in the CAP rhythm was observed between experimental and control eyes. At concentrations from 3 to $6 \mathrm{~mm}$, the cycles of CAP activity were often reduced in magnitude, though circadian rhythmicity was still evident. No spiking activity was evident during treatments 

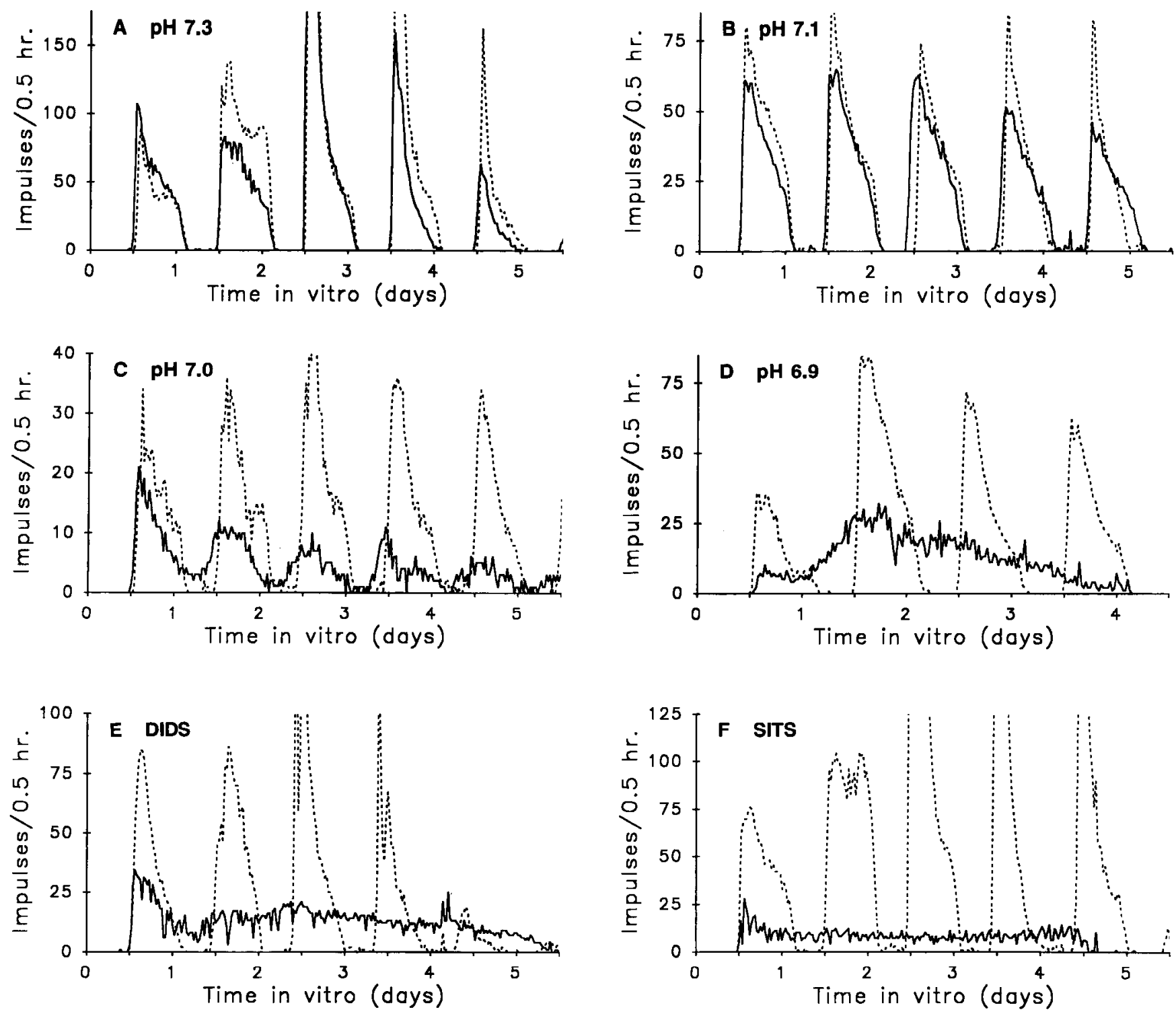

Figure 1. Plots of CAP activity from eyes exposed to continuous treatments in constant darkness. In all plots, the solid trace is the experimental eye, and the broken trace, the untreated control eye from the same animal. Impulse frequency in impulses per $0.5 \mathrm{hr}$ is plotted against time of the preparation in vitro. Time 0 is subjective dusk (CT 12) relative to the previous light/dark cycle in vivo. Tick marks on the abscissa occur every 12 $\mathrm{hr}$; continuous treatments were applied $1 \mathrm{hr}$ after time 0 (CT 13). $A-D$, ASW at pH 7.3, 7.1, 7.0, and 6.9, respectively; $E$, $30 \mu \mathrm{M}$ DIDS in $0.05 \%$ DMSO; $F, 50 \mu \mathrm{M}$ SITS in $0.05 \%$ DMSO.

at concentrations of $7 \mathrm{~mm}$ and higher (see experiments below) at least over the first cycle.

\section{Effects of long-duration pulse treatments}

An absence of circadian rhythmicity suggests that either the pacemaker is no longer oscillating, or the CAP activity is no longer driven by the pacemaker. To test for the possibility that the underlying circadian pacemaker is affected by low intracellular $\mathrm{pH}$, we employed an experimental paradigm previously uscd by Pittendrigh (1960). In this scheme, pulses all begin at the same circadian time but terminate at different times and range in duration from 4 to $48 \mathrm{hr}$. The timing of the phase reference points of the rhythm following the experimental pulses will indicate the status of the pacemaker during the treatment. For example, if the pacemaker has been uncoupled from its output but has continued its motion, then the phase of the rhythm subsequent to each experimental pulse should bear a strong relationship to the expected phase of untreated control eyes.

This paradigm was applied using ASW at $\mathrm{pH}$ 6.8. An example of a long-duration pulse of ASW at pH 6.8 is shown in Figure $2 A$. The pulse was applied to the experimental eye (solid trace) from CT 13 to 12 (23 hr in length) over the first cycle. The phase of the rhythm subsequent to the treatment has shifted substantially relative to the untreated control eye rhythm (broken trace), suggesting that the underlying circadian pacemaker, and not just the overt rhythm, is sensitive to the treatment. Expressed as a delay relative to the control eye rhythm on the fourth cycle, the phase shift was $-15.5 \mathrm{hr}$. This record is typical of all of the long-duration pulse treatments in that (1) no spiking was observed during the $\mathrm{pH} 6.8$ treatment; (2) the first peak following the treatment, especially for longer pulses, appeared 

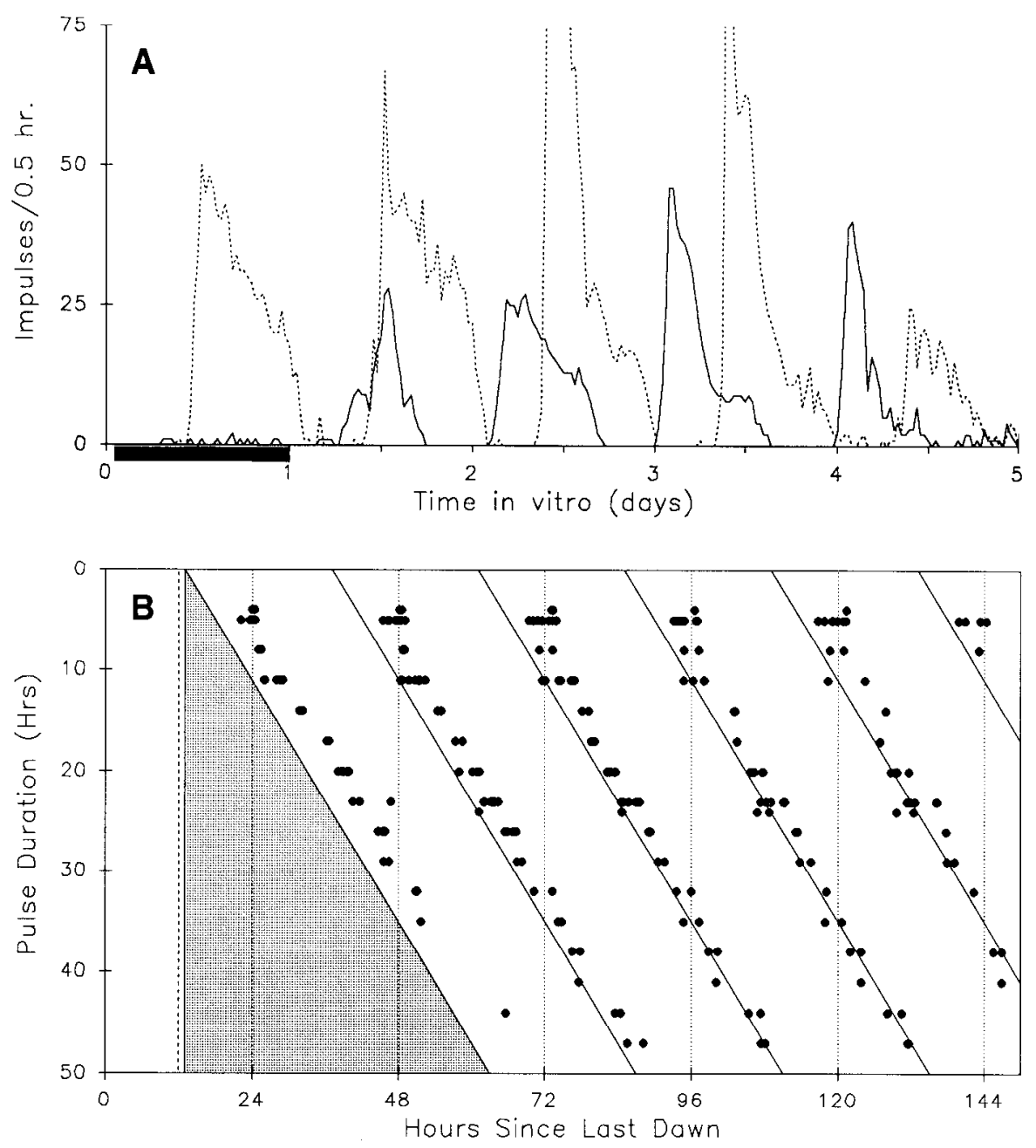

Figure 2. A, CAP activity record for an experiment with a pulse treatment at pH 6.8 from CT 13 to 12 , indicated by the solid bar below the horizontal time axis. The plotting convention is as in Figure 1. There is a substantial shift in phase following the treatment relative to the control eye rhythm. $B$, Phase points of circadian cycles of activity (horizontal axis) for up to $6 \mathrm{~d}$ are plotted as a function of the length of treatment (vertical axis) with ASW at $\mathrm{pH}$ 6.8. Time 0 on the horizontal axis represents subjective dawn of the last cycle of activity before the eyes are placed in vitro at $\mathrm{CT} 12$, indicated by the vertical dashed line. All treatments began at CT 13 and continued from 4 up to $47 \mathrm{hr}$, as indicated by the triangular shaded region. Treatments that would not perturb the pacemaker would yield phase points that would line up approximately at subjective dawn, as indicated by the vertical dotted lines. The solid diagonal reference lines represent phases that are a multiple of $24 \mathrm{hr}$ following the end of the treatment. Phase points for pulses up to the first subjective dawn are close to subsequent subjective dawns, whereas all subsequent phase points following longer treatments yield phase points that are a function of the ending time of the low-pH treatment. reduced in magnitude; and (3) the phase difference between control and experimental rhythms was more delayed on the first cycle following the treatment than on subsequent cycles (see below).

The results of the experiment with this paradigm using the full range of pulse lengths support the hypothesis that the pacemaker has been stopped. In Figure $2 B$, the phases of the peaks of activity for all treated eyes are plotted on the horizontal axis, as hours since last dawn (CT 24), versus the vertical axis, which shows duration of the pulse of $\mathrm{pH}$ 6.8. Pulse-treatment timing and duration are indicated by the shaded triangle. All pulses began at CT 13, 1 hr after the eyes were set up in vitro (CT 12). Pulse length ranged from 4 to $47 \mathrm{hr}$, mostly in 3-hr increments. Untreated control eye rhythms show phases (data not shown) that line up with subsequent projected dawns (vertical dotted lines). However, subsequent phases of low-pH-treated eye rhythms (at least for pulses longer than $8 \mathrm{hr}$ in length; see below) were a function of the circadian time of the end of the pulse, as indicated by the solid diagonal reference lines (Fig. 2B).

A comparison of the timing of phase reference points of only the first and second cycles of activity following the experimental pulse in Figure $2 B$ might suggest that the period of the experimental rhythms may be substantially shorter than that expected from untreated control eyes (see also Fig. 2A). However, a comparison of the timing of the phases of the third, fourth, and fifth cycles indicates that the period is, in fact, stable and similar to that expected for untreated control eyes. The first two cycles following treatment appear to exhibit "transient" phases, before a stable phase of the rhythm is expressed in subsequent cycles.

Prcliminary cxperiments on the Aplysia ocular pacemaker suggest that low extracellular $\mathrm{pH}$ treatment is also effective in that system. A 23-hr pulse of $\mathrm{pH} 6.8$ ASW delivered to Aplysia eyes from CT 13 to $12(N=2)$ yielded small but stable phase advances ( $1.8 \mathrm{hr}$ in one eye as measured on the fourth cycle; in the other eye, the low amplitude of its fourth cycle was too low to meet criteria - the phase shift measured on the third cycle was $1.3 \mathrm{hr}$ ). This result, together with the observation that CAP activity in the Aplysia eyes was not completely inhibited during the $\mathrm{pH} 6.8$ treatment, suggested that the $\mathrm{pH}$ sensitivity of the Aplysia pacemaker to low extracellular $\mathrm{pH}$ may be less than that of the Bulla pacemaker. To test this possibility, pulses of ASW at pH 6.0 were applied from CT 13 to $12(N=2)$. In this case, CAP activity was completely inhibited during the treatment, and substantial phase shifts (phase delays of $-15.0 \mathrm{hr}$ and -14.7 hr) were observed. These results are similar to those in Bulla with pulses of ASW at $\mathrm{pH} 6.8$.

\section{Phase versus duration dependency}

The absence of phase shifts following the $\mathrm{pH} 6.8$ pulses from CT 13 to 17 , from CT 13 to 18 , and from CT 13 to 21 (pulse durations of 4,5 , and $8 \mathrm{hr}$ ) in Figure $2 B$ suggests the possibility of either phase dependence (a critical phase at CT 24 must be 


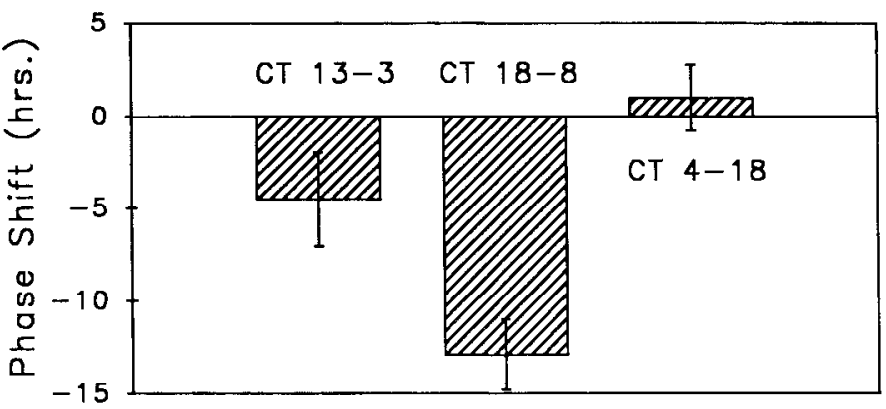

Figure 3. Average phase shifts with $95 \%$ confidence intervals measured on the fourth cycle to pulses of $\mathrm{pH} 6.8 \mathrm{ASW}$ delivered from CT 13 to 3 , CT 18 to 8 , and CT 4 to $18(N=4$ each $)$ over the first cycle of activity. Delay phase shifts are plotted as negative values. Averages with $95 \%$ confidence intervals are as follows: $C T 13$ to $3,-4.5 \mathrm{hr} \pm$ 2.6; CT 18 to $8,-12.9 \mathrm{hr} \pm 1.9 ;$ CT 4 to $18,+1.0 \mathrm{hr} \pm 1.8$.

spanned) or duration dependence (a critical, minimum pulse duration of about $11 \mathrm{hr}$ must be applied) of the low-pH treatment. To distinguish between these two possibilities, we have applied $\mathrm{pH} 6.8$ pulses of $14 \mathrm{hr}$ duration at different phases during the cycle. The phase shifts to pulses delivered from CT 13 to 3 , CT 18 to 8 , and CT 4 to 18 were measured on the fourth cycle relative to untreated control eyes; the average phase shifts are plotted in Figure 3. Because the magnitude of the phase shifts is significantly different (ANOVA, $p<0.0005$ ) at different phases, these data favor phase dependency over duration dependency.

\section{Salicylate sensitivity}

Pulses of the sodium salt of the weak acid salicylate, which lowers intracellular $\mathrm{pH}$ independently of extracellular $\mathrm{pH}$ (Sharp and Thomas, 1981), were also capable of shifting the phase substantially. In Figure $4 A$, a pulse of $15 \mathrm{~mm}$ salicylate applied from CT 13 to 12 yields a large phase shift of the experimental eye $(-9.9 \mathrm{~h})$. To examine further the dose dependence of intracellular $\mathrm{pH}$, we applied pulses of salicylate at various concentrations from CT 13 to 12 over the first cycle and measured phase shifts relative to controls on the fourth cycle. The results of this experiment appear in Figure $4 B$. At $8 \mathrm{~mm}$ there is a sharp transition in phase shift magnitudes, from the small phase shifts, mostly advances, observed at concentrations less than $8 \mathrm{~mm}$ (range of $-0.2 \mathrm{hr}$ to $+3.9 \mathrm{hr}$ ), to the large phase delays observed at concentrations higher than $8 \mathrm{~mm}$ (range of -7.7 to -11.9 hr). At the critical 8-mm concentration, one eye exhibited the large delay characteristic of higher salicylate concentrations $(-9.3$ $\mathrm{hr}$ ), whereas two eyes exhibited small shifts characteristic of lower concentrations $(-1.1 \mathrm{hr},+1.8 \mathrm{hr})$.

\section{Discussion}

Two treatments used to lower intracellular $\mathrm{pH}$ yielded noncircadian CAP activity: lowering extracellular $\mathrm{pH}$ to 6.9 , and application of the acid extrusion inhibitors DIDS and SITS.

Despite the ubiquitous regulation of intracellular $\mathrm{pH}$ (Roos and Boron, 1981), manipulation of extracellular $\mathrm{pH}$ in an effort to overcome the regulating mechanism leads to changes in intracellular $\mathrm{pH}$ levels, though the rate and magnitude of these changes are low (Thomas, 1974; Roos and Boron, 1981; Nuccitelli and Heiple, 1982; Busa and Nuccitelli, 1984; Drapeau and Nachshen, 1988). In isolated presynaptic nerve terminals from rat brain, a drop in extracellular $\mathrm{pH}$ from 7.4 to 5.5 pro-
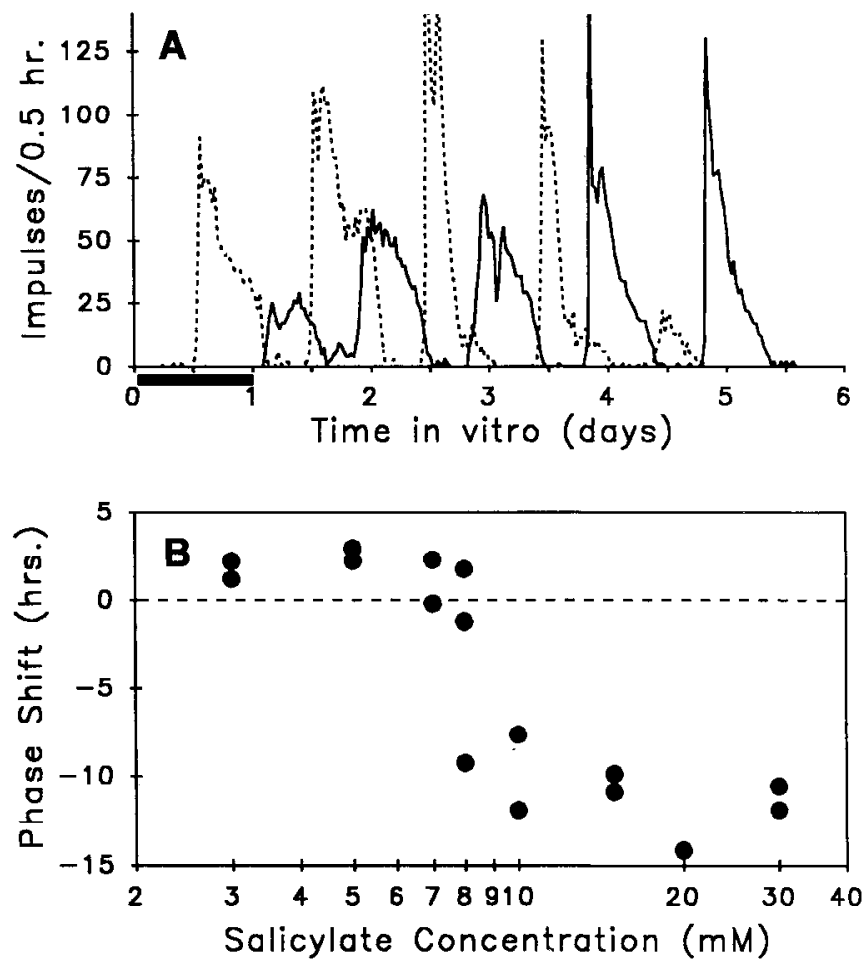

Figure 4. A, CAP activity records for a pulse treatment of $15 \mathrm{~mm}$ salicylate from CT 13 to 12 , indicated by the solid bar below the horizontal time axis. There is a substantial phase shift following the treatment relative to the control eye, as observed with ASW at pH 6.8 (cf. Fig. $2 A$ ). $B$, Dose-response curve for phase shifts to salicylate pulses delivered from CT 13 to 12 . For concentrations at $8 \mathrm{~mm}$ and higher, phase shifts are plotted as phase delays (negative values on the vertical axis). Note the sharp transition in phase-shift magnitude at the critical $8 \mathrm{~mm}$ concentration.

duced a "slow" decrease in intracellular pH from 7.2 to a steadystate level of about 5.8 as measured by a $\mathrm{pH}$-sensitive fluorescent indicator (Drapeau and Nachshen, 1988). In snail neurons monitored with a pH-sensitive intracellular microelectrode, the avcrage intracellular $\mathrm{pH}$ was 7.4. A drop in extracellular $\mathrm{pH}$ from 8.0 to 7.0 yielded an intracellular $\mathrm{pH}$ decrease of 0.1 units over a 10-min period, with the rate of decrease being fastest at the beginning of the $\mathrm{pH}$ drop. With an extracellular $\mathrm{pH}$ of 6.0 , "internal $\mathbf{p H}$ decreased at rates which increased during the period of exposure, suggesting that the membrane permeability was significantly changed. The change in internal $\mathrm{pH}$, however, was still relatively slow." (Thomas, 1974). For the purposes of a circadian study in which the time scale of treatments is long, changing extracellular $\mathrm{pH}$ serves as a simple but reliable manipulation of intracellular $\mathrm{pH}$. However, given the variability of response of different cell types to extracellular $\mathrm{pH}$ (Nuccitelli and Heiple, 1982), the degree of decrease in intracellular $\mathrm{pH}$ with our lowered extracellular $\mathrm{pH}$ treatments remains unclear. Furthermore, complete proof of the involvement of intracellular $\mathrm{pH}$ as the effective variable in this study must await a direct measurement of intracellular $\mathrm{pH}$ under the conditions applied.

The extracellular $\mathrm{pH}$ of the bathing ASW clearly affects the expression of the circadian rhythm. At extracellular $\mathrm{pH}$ of 7.0 and higher, the circadian rhythm is still apparent; however, the amplitude of the rhythm is degraded with decreasing $\mathrm{pH}$, as though the rhythm is being "damped out" (Fig. 1A-C). At an extracellular $\mathrm{pH}$ of 6.9 , the absence of circadian rhythmicity in 
Bulla is apparent (Fig. $1 D$ ). The possibility that extracellular $\mathrm{pH}$ itself might be the critical affected variable was addressed by applying treatments that lower intracellular $\mathrm{pH}$ independently of extracellular pH. Our results, discussed below, with DIDS and SITS continuously applied and with salicylate applied in pulses are consistent with results obtained with equivalent treatments using low extracellular $\mathrm{pH}$.

Intracellular $\mathrm{pH}$ is actively regulated to a more basic value than would be predicted from a passive distribution of the hydrogen ion across the cell membrane (Thomas, 1974; Roos and Boron, 1981). Therefore, inhibitors of the $\mathrm{pH}$-regulating mechanism should lower intracellular $\mathrm{pH}$ by inhibiting acid extrusion. In mesangial cells in which an SITS-sensitive mechanism is involved in $\mathrm{pH}$ regulation, SITS application leads to intracellular acidification (Ganz et al., 1989). In invertebrate neurons such as the squid giant axon (Boron and Russell, 1983) and snail neuron (Thomas, 1977, 1982), the primary $\mathrm{pH}$-regulating mechanism, an anion transporter, is also inhibited by the stilbene derivatives SITS and DIDS. The ability of SITS and DIDS to generate arrhythmicity in this study is consistent with their action in lowering intracellular $\mathrm{pH}$. However, caution is necessary in interpreting results with the stilbene derivatives; though they do effectively inhibit pH-regulating membrane transport systems, they are also highly unspecific compounds (W. F. Boron, personal communication) and may be affecting the circadian pacemaker in an unknown manner.

Weak acids applied extracellularly are known to lower intracellular pH reliably (Roos and Boron, 1981). The weak acid salicylate was chosen. In a comparative study of the effectiveness of various weak acids at lowering the intracellular $\mathrm{pH}$ of $\mathrm{crab}$ muscle, Sharp and Thomas (1981) reported that the weak acid salicylate produced the largest decrease in intracellular $\mathrm{pH}(100$ $\mathrm{mm}$ salicylate lowered intracellular $\mathrm{pH}$ by $0.6 \mathrm{pH}$ units). The ability of salicylate pulses at concentrations greater than $8 \mathrm{~mm}$ to generate large phase shifts comparable to those obtained with $\mathrm{pH} 6.8$ application is consistent with the effectiveness of salicylate in lowering intracellular $\mathrm{pH}$.

The failure to observe arrhythmic CAP activity with continuous treatment at lower salicylate concentrations might be accounted for if salicylate was exerting inhibitory effects on spiking activity at those concentrations effective at generating arrhythmicity. In fact, salicylate is known to affect membrane excitability in several preparations (Neto and Narahashi, 1976; Attwell et al., 1979) including molluscan neurons (Barker and Levitan, 1974). Furthermore, other reported intracellular side effects of salicylate such as inhibition of oxidative phosphorylation (Brody, 1956) or prostaglandin synthesis (Ferreira and Vane, 1974) could be interfering with the expression of CAP. activity, as well as with phasc-shifting cffects. On the other hand, other weak acids do appear to lead to noncircadian CAP activity, at least in the Aplysia ocular circadian rhythm. Jacklet (1976) has previously reported that CAP activity is devoid of circadian modulation in the presence of acetate, propionate, and butyrate, which are all weak acids known to be effective at lowering intracellular pH (Roos and Boron, 1981; Sharp and Thomas, 1981). Preliminary experiments in this study support the sensitivity of the Aplysia pacemaker to low $\mathrm{pH}$ pulses, though this sensitivity may be less than that in Bulla.

An observed absence of circadian rhythmicity in Bulla suggests that either the underlying circadian pacemaker has been affected (has stopped or become arrhythmic), or the CAP activity output has been "uncoupled" from the pacemaker (only the pacemaker's modulation of CAP activity has been interrupted). The observation that the subsequent phase of the circadian rhythm is tightly correlated with the phase of the end of the low-pH pulses (Fig. $2 B$ ), at least for pulses longer than $8 \mathrm{hr}$, suggests that it is the pacemaker itself that is affected by the low intracellular $\mathrm{pH}$. In this view, the pacemaker is held motionless during the low-pH treatment. After normal $\mathrm{pH}$ is restored, the pacemaker resumes its motion, which is now phase delayed with respect to an untreated control eye rhythm by an amount related to the duration of the low-pH pulse.

The three shortest pulses in Figure $2 B$ yielded phases no different than would be expected for untreated eye rhythms. To account for this observation, two possibilities must be considered.

(1) The low pH treatment must be applied over a critical phase of the cycle in order for pacemaker motion to be stopped, and the pacemaker can be stopped only at this phase of the cycle. From Figure $2 B$, this critical phase would be very close to subjective dawn (CT 24), because the phase reference points do not line up with the diagonal reference lines until the pulses end at or after this time.

(2) A minimum-duration pulse is required in order for pacemaker motion to be stopped, and the pacemaker can be stopped at any phase. From Figure $2 B$, this critical duration would appear to be at least $11 \mathrm{hr}$, because the first pulse yielding phases different from dawn is $14 \mathrm{hr}$ in length.

The observed magnitude of the phase shifts in response to three different 14-hr pulses (Fig. 4) is consistent with a criticalphase hypothesis. If a minimum pulse duration was involved, then all 14-hr pulses should have yielded similar phase shifts irrespective of the phase at which they were applied. Furthermore, the magnitudes of the observed phase shifts are consistent with a phase dependence with a critical phase near dawn (CT 24). No phase shift occurred following the CT 4 to 18 pulse (the pulse did not cross the critical phase at CT 24), and the largest phase shift occurred following the CT 18 to 8 pulse (CT 24 was crossed early in the pulse). The location of the critical phase at dawn is further supported by the observation that CAP activity begins normally at subjective dawn on the first cycle with continuous treatments of $\mathrm{pH}$ 6.9, SITS, and DIDS (Fig. 1).

Overall, these data suggest that circadian pacemaker motion is stopped in a phase-dependent manner by treatments applied to lower intracellular $\mathrm{pH}$. It is of interest to compare the effects of low $\mathrm{pH}$ with other treatments such as constant light, which has also been shown to abolish circadian rhythmicity in some circadian systems. Following the termination of constant light for at least $12 \mathrm{hr}$ duration, the phase of the subsequent restored circadian rhythm has been observed to begin at CT 12 , suggesting that the pacemaker was stopped in constant light (Pittendrigh, 1960, 1966; Saunders, 1976; Prichard and Lickey, 1981). However, Pittendrigh (1981), using lower-intensity constant light, still observed that the rhythm subsequent to light treatment began at CT 12, despite evidence of circadian rhythmicity during the light treatment (Pittendrigh, 1981). Furthermore, subsequent phases following long, constant light pulses (in an experiment similar to that in Fig. $2 B$ ) have been observed to vary in a circadian fashion as a function of light-pulse duration (Saunders, 1976). These two observations have led to suggestions that the underlying pacemaker may actually continue its motion in constant light, and that the transition from constant light to dark instantaneously "resets" the pacemaker to $\mathrm{CT} 12$. 
It is therefore necessary to consider whether the pacemaker is "resetting" to CT 24 at the end of a low-pH pulse, rather than stopping its motion at that phase during the pulse. Although we cannot conclusively rule out this possibility, several observations suggest that low $\mathrm{pH}$ affects the pacemaker differently than constant light: (1) no phase dependency has been reported for constant light, and it must be applied for $12 \mathrm{hr}$ in order to reset the pacemaker, whereas low-pH pulses must include the sensitive phase at dawn, and their effectiveness appears to be duration independent; (2) if low pH is resetting, then it is doing so at CT 24 rather than CT 12; and (3) we have observed no circadian variability in subsequent phases with increased pulse duration (Fig. 2B). These differences between constant light and low $\mathrm{pH}$ lead us to favor the view that the pacemaker is stopping in the presence of low $\mathrm{pH}$. The data in this study are very similar to the phase-dependent effect on the circadian pacemaker in Drosophila in response to inhibition of aerobic metabolism, which has not been described as a resetting phenomenon (Pittendrigh, 1974).

The mechanism by which low intracellular $\mathrm{pH}$ affects the pacemaker is unknown. Inhibition of protein synthesis has been tightly correlated with lower intracellular $\mathrm{pH}$ values in sperm and egg activation and in the cell cycle (Nuccitelli and Heiple, 1982; Winkler, 1982; Busa and Nuccitelli, 1984). Furthermore, an autonomous intracellular $\mathrm{pH}$ oscillator with a period of 4$5 \mathrm{hr}$ has been reported in Dictyostelium cells, which may time the cell cycle by accelerating protein and DNA synthesis (Aerts et al., 1985). A recent study in Bulla suggests that protein synthesis inhibition is capable of stopping the motion of the circadian pacemaker in a phase-dependent manner, and that the critical phase is near subjective dawn (Khalsa and Block, 1990b). The involvement of protein synthesis in the pacemaker mechanism is further suggested by reported circadian changes in protein levels in the Aplysia ocular pacemaker (Strumwasser, 1987; A. Eskin, personal communication). These data are consistent with inhibition of protein synthesis as a potential mechanism by which low $\mathrm{pH}$ affects the pacemaker.

It is conceivable that low $\mathrm{pH}$ is affecting pacemaker proteins directly. The phase-shifting versus concentration data with salicylate (Fig. 4) suggests the possibility that the $\mathrm{pH}$ sensitivity of the pacemaker undergoes an abrupt transition over a narrow range. This is consistent with the observation that the circadian rhythm is abolished over a narrow extracellular $\mathrm{pH}$ range of 0.1 unit (from 7.0 to 6.9 ; Fig. $1 C, D$ ), though confirmation of the actual intracellular $\mathrm{pH}$ changes with these treatments requires a direct measurement of the intracellular $\mathrm{pH}$. If there is a corresponding steep intracellular $\mathrm{pH}$ dependence of the pacemaker, this may reflect the $\mathrm{pH}$ dependence of a protein element within the central mechanism of the pacemaker. The activities of the enzymes calmodulin, phosphofructokinase, adenylyl cyclase, and cyclic nucleotide phosphodiesterase all exhibit a pronounced sensitivity to intracellular $\mathrm{pH}$ (Roos and Boron, 1981; Nuccitelli and Heiple, 1982; Busa and Nuccitelli, 1984; Busa, 1986; Green and Gillette, 1988).

The relationship between intracellular $\mathrm{pH}$ and intracellular calcium may be especially relevant to the Bulla pacemaker, because calcium serves as a second messenger in mediating depolarization-induced phase shifts (Khalsa and Block, 1988), and because reductions in spontaneous transmembrane calcium fluxes appear to induce phase shifts (Khalsa and Block, 1990a). Furthermore, Raju et al. (1990a,b) have reported changes in the level of a calcium-binding protein associated with phase-shifting pulses in the Aplysia pacemaker. Calcium loading and release from sarcoplasmic reticulum, $\mathrm{Ca}-\mathrm{H}$ exchange, intracellular calcium levels, calcium fluxes, and transport have all been reported as pH dependent (Roos and Boron, 1981; Busa and Nuccitelli, 1984; Busa, 1986; Drapeau and Nachshen, 1988).

It is possible that a rhythm in intracellular $\mathrm{pH}$ serves a central active role in the pacemaker system, though it is also possible that some variable within the pacemaker exhibits a sharp $\mathrm{pH}$ dependence at the critical phase at dawn. In the latter case, intracellular $\mathrm{pH}$ would perform an obligatory supportive role as a nonrhythmic passive element-it must be regulated at appropriate levels within the cell in order for circadian oscillation to proceed.

\section{References}

Aerts RJ, Durston AJ, Moolenaar WH (1985) Cytoplasmic pH and the regulation of the Dictyostelium cell cycle. Cell 43:653-657.

Attwell D, Bergman C, Ojeda C (1979) The action of salicylate ions on the frog node of Ranvier. J Physiol (Lond) 295:69-81.

Barker JL, Levitan H (1974) Studies on mechanisms underlying nonnarcotic analgesia. Adv Neurol 4:503-510.

Block GD, Wallace S (1982) Localization of a circadian pacemaker in the eye of a mollusc, Bulla. Science 217:155-157.

Boron WF, Russell JM (1983) Stoichiometry and ion dependencies of the intracellular-pH-regulating mechanism in squid giant axons. $J$ Gen Physiol 81:373-399.

Brody TM (1956) Action of sodium salicylate and related compounds on tissue metabolism in vitro. J Pharmacol Exp Ther 117:39-51.

Busa WB (1986) Mechanisms and consequences of pH-mediated cell regulation. Annu Rev Physiol 48:389-402.

Busa WB, Nuccitelli R (1984) Metabolic regulation via intracellular pH. Am J Physiol 246:R409-R438.

Drapeau P, Nachshen DA (1988) Effects of lowering extracellular and cytosolic pH on calcium fluxes, calcium levels, and transmitter release in presynaptic nerve terminals isolated from rat brain. J Gen Physiol 91:305-315.

Ferreira SH, Vane JR (1974) New aspects of the mode of action of non-steroid anti-inflammatory drugs. Annu Rev Pharmacol 14:5773.

Ganz MB, Boyarsky G, Sterzel RB, Boron WF (1989) Arginine vasopressin enhances $\mathrm{pH}_{i}$ regulation in the presence of $\mathrm{HCO}_{3}^{-}$by stimulating three acid-base transport systems. Nature 337:648-651.

Green DJ, Gillette R (1988) Regulation of cAMP-stimulated ion current by intraccllular $\mathrm{pH}, \mathrm{Ca}^{2+}$, and calmodulin blockers. J Neurophysiol 59:248-258.

Jacklet JW (1976) Circadian rhythms in the nervous system of a marine gastropod, Aplysia. In: Biological rhythms in the marine environment (DeCoursey PJ, ed), pp 17-31. Columbia, SC: University of South Carolina.

Jacklet JW (1984) Neural organization and cellular mechanisms of circadian pacemakers. Int Rev Cytol 89:251-294.

Jacklet JW (1989) Circadian neuronal oscillators. In: Neuronal and cellular oscillators (Jacklet JW, ed), pp 483-527. New York: Dekker.

Khalsa SBS, Block GD (1988) Calcium channels mediate phase shifts of the Bulla circadian pacemaker. J Comp Physiol 164:195-206.

Khalsa SBS, Block GD (1990a) Calcium in phase control of the Bulla circadian pacemaker. Brain Res 506:40-45.

Khalsa SBS, Block GD (1990b) Low pII and protein synthesis inhibitors apparently stop the motion of a neuronal circadian pacemaker. Soc Neuorsci Abstr 16:640.

McMahon DG, Block GD (1987) The Bulla circadian pacemaker. I: Pacemaker membrane potential regulates phase through a calcium dependent mechanism. J Comp Physiol 161:335-346.

McMahon DG, Wallace SF, Block GD (1984) Cellular analysis of the Bulla ocular circadian pacemaker system. II: Neurophysiological basis of circadian rhythmicity. J Comp Physiol 155:379-385.

Neto FR, Narahashi T (1976) Ionic mechanism of the salicylate block of nerve conduction. J Pharmacol Exp Ther 199:454-463.

Nuccitelli R, Heiple JM (1982) Summary of the evidence and discussion concerning the involvement of $\mathrm{pH}_{i}$ in the control of cellular functions. In: Intracellular pH: its measurement, regulation and uti- 
lization in cellular functions (Nuccitelli R, Deamer DW, eds), pp 567586. New York: Liss.

Pittendrigh CS (1960) Circadian rhythms and the circadian organization of living systems. Cold Spring Harbor Symp Quant Biol 25: 159-184.

Pittendrigh CS (1966) The circadian oscillation in Drosophila pseudoobscura pupae: a model for the photoperiodic clock. Z Pflanzenphysiol 54:275-307.

Pittendrigh CS (1974) Circadian oscillations in cells and the circadian organization of multicellular systems. In: The neurosciences, 3rd study program (Schmidt FO, Worden FG, eds), pp 437-458. Cambridge, MA: MIT Press.

Prichard RG, Lickey ME (1981) In vitro resetting of the circadian clock in the Aplysia eye. J Neurosci 1:835-839.

Raju U, Yeung SJ, Eskin A (1990a) Involvement of proteins in light resetting ocular circadian oscillators of Aplysia. Am J Physiol 258: R256-R262.

Kaju U, Nunez-Kegueiro M, Cook R, Eskin A (1990b) Characterization of a putative circadian oscillator protein in the eye of Aplysia. Soc Neurosci Abstr 16:1332.

Ralph MR, Block GD (1990) Circadian and light-induced conductance changes in putative pacemaker cells of Bulla gouldiana. J Comp Physiol 166:589-595.

Roos A, Boron WF (1981) Intracellular pH. Physiol Rev 61:296-434.

Saunders DS (1976) The circadian eclosion rhythm in Sarcophaga argyrostoma: some comparisons with the photoperiodic "clock." J Comp Physiol 110:111-133.
Sharp AP, Thomas RC (1981) The effects of chloride substitution on intracellular $\mathrm{pH}$ in crab muscle. J Physiol (Lond) 312:71-80.

Strumwasser F (1987) Circadian oscillators in molluscs and other organisms: mechanisms and relevance for neuropsychiatry. In: Neurobiology, molluscan models (Boer HH, Geraerts WPM, Joosse J, eds), pp 298-310. Amsterdam: North-Holland.

Takabashi JS, Murakami N, Nikaido SS, Pratt BL, Robertson LM (1989) The avian pineal, a vertebrate model system of the circadian oscillator: cellular regulation of circadian rhythms by light, secondary messengers and macromolecular synthesis. Horm Res 45:279-351.

Thomas RC (1974) Intracellular $\mathrm{pH}$ of snail neurones measured with a new pH-sensitive glass micro-electrode. J Physiol (Lond) 238:159180.

Thomas RC (1977) The role of bicarbonate, chloride and sodium ions in the regulation of intracellular $\mathrm{pH}$ in snail neurones. J Physiol (Lond) 273:317-338.

Thomas RC (1982) Snail neuron intracellular pH regulation. In: Intracellular $\mathrm{pH}$ : its measurement, regulation and utilization in cellular functions (Nuccitelli R, Deamer DW, eds), pp 189-204. New York: Liss.

Winkler MM (1982) Regulation of protein synthesis in sea urchin eggs by intracellular $\mathrm{pH}$. In: Intracellular $\mathrm{pH}$ : its measurement, regulation and utilization in cellular functions (Nuccitelli R, Deamer DW, eds), pp 325-340. New York: Liss. 